\title{
Unidominating Functions of Corona Product Graph $P_{n} \odot W_{1, m}$
}

\author{
B. Aruna \\ Department of Mathematics, \\ Kallam Haranadha Reddy Institute of Technology, \\ Guntur - 522019, Andhra Pradesh, India
}

\author{
B. Maheswari \\ Department of Applied Mathematics, \\ Sri Padmavati Mahia Visvavidyalayam, \\ Tirupati - 517502, Andhra Pradesh, India
}

\begin{abstract}
Graph Theory is the developing area of research and it is one of the important branch of Mathematics. The theory of domination in graphs introduced by Ore [7] and Berge [2] is a fascinating area of research in graph theory and it has witnessed a spectacular growth in the last three decades.

Corona product graph is a new concept introduced by Frucht and Harary [3] and it is receiving much attention of the researchers. A new product on two graphs $G_{1}$ and $G_{2}$, called corona product denoted by $G_{1} \odot G_{2}$. The object is to construct a new and simple operation on two graphs $G_{1}$ and $G_{2}$ called their corona, with the property that the group of the new graph is in general isomorphic with the wreath product of the groups of $G_{1}$ and of $G_{2}$.

In this paper the authors have studied the concept of unidominating function and upper unidomination number for corona product graph $P_{n} \odot W_{1, m}$ and determined the unidomination number and upper unidomination number for $P_{n} \odot W_{1, m}$. Also the number of unidominating functions of minimum weight is found.
\end{abstract}

\section{Keywords}

Unidominating function, unidomination number, minimal unidominating function, upper unidomination number.

\section{INTRODUCTION}

In recent years dominating functions in domination theory playing a key role as they have interesting applications. It has been studied extensively and finds wide applications to various branches of Science \& Technology. The concepts of dominating functions are introduced by Hedetniemi [4]. Domination and its properties have been extensively surveyed and studied detailed by in T.W. Haynes and others [5, 6].

A new product on two graphs $G_{1}$ and $G_{2}$, called corona product denoted by $\mathrm{G}_{1} \odot \mathrm{G}_{2}$ was introduced by Frucht and Harary [3] and it has become an interesting area of research at present.

Anantha Lakshmi [1] has introduced new concepts unidomination, upper unidomination, minimal unidominating function of a graph and studied these functions for some standard graphs.

The authors have studied the concepts unidominating function and upper unidomination number for corona product graph $\mathrm{P}_{\mathrm{n}} \odot \mathrm{W}_{1, \mathrm{~m}}$. Also the unidomination and upper unidomination number of the above said graph is found. Further, the number of unidominating functions of minimum weight and minimal unidominating functions of maximum weight for this graph are determined.

\section{CORONA PRODUCT OF $P_{n}$ AND $W_{1, m}$}

The corona product of a path $P_{n}$ with a wheel graph $W_{1, m}$ is a graph obtained by taking one copy of a $n$ - vertex graph $P_{n}$ and $n$ copies of $W_{1, m}$ and then joining the $i^{t h}$ - vertex of $P_{n}$ to every vertex of $i^{t h}$ - copy of $W_{1, m}$. This is denoted by $P_{n} \odot W_{1, m}$

The vertices in $P_{n}$ are denoted by $v_{i}$, the centre and the vertices of the wheel $W_{1, m}$ are denoted respectively by $u_{i}$ and $u_{i j}$.

\section{UNIDOMINATION NUMBER OF $P_{\mathbf{n}} \odot \mathbf{W}_{1, \mathrm{~m}}$}

In this section the concepts of unidominating function, unidomination number are defined. The unidomination number and the number of unidominating functions of minimum weight of $P_{n} \odot W_{1, m}$ are determined.

Definition 1: Let $G(V, E)$ be a graph. A function $f: V \rightarrow$ $\{0,1\}$ is said to be a unidominating function if

$$
\begin{gathered}
\sum_{u \in N[v]} f(u) \geq 1 \quad \forall v \in V \text { and } f(v)=1, \\
\sum_{u \in N[v]} f(u)=1 \quad \forall v \in V \text { and } f(v)=0 .
\end{gathered}
$$

where $N[v]$ is the closed neighbourhood of the vertex $v$.

Definition 2: The unidomination number of a graph $G(V, E)$ is defined as

$\min \{f(V) / f$ is a unidominating function $\}$.

It is denoted by $\gamma_{u}(G)$.

Here $f(V)$

$=\sum_{u \in V} f(u)$ is called as the weight of the unidominating function $f$.

Theorem 3.1: The unidomination number of $P_{n} \odot W_{1, m}$ is $n$.

Proof: Let $P_{n} \odot W_{1, m}$ be the given corona product graph.

To find the unidomination number of $P_{n} \odot W_{1, m}$ the following cases arise.

Case 1: Define a function $f: V \rightarrow\{0,1\}$ by

$f\left(v_{i}\right)=1$ for $i=1,2, \ldots \ldots, n$,

$f\left(u_{i}\right)=0$ for $i=1,2, \ldots \ldots, n$

and $f\left(u_{i j}\right)=0$ for $i=1,2, \ldots \ldots, n$ and $j=1,2, \ldots \ldots, m$. 
Now we show that $f$ is a unidominating function.

Let $i \neq 1$ and $i \neq n$.

If $v_{i} \in P_{n}$ then

$$
\begin{aligned}
& \sum_{u \in N\left[v_{i}\right]} f(u)=f\left(v_{i-1}\right)+f\left(v_{i}\right)+f\left(v_{i+1}\right)+f\left(u_{i}\right)+f\left(u_{i 1}\right) \\
&+ f\left(u_{i 2}\right)+\cdots+f\left(u_{i m}\right) \\
&=1+1+1+0+0+0+\cdots+0=3 .
\end{aligned}
$$

If $u_{i} \in W_{1, m}$ then

$$
\begin{aligned}
\sum_{u \in N\left[\mathrm{u}_{i}\right]} f(u)=f\left(v_{i}\right) & +f\left(u_{i}\right)+f\left(u_{i 1}\right)+f\left(u_{i 2}\right)+\cdots \\
& +f\left(u_{i m}\right)=1+0+0+0+\cdots+0 \\
& =1 .
\end{aligned}
$$

If $u_{i j} \in W_{1, m}$ then

$$
\begin{gathered}
\sum_{u \in N\left[u_{i j}\right]} f(u)=f\left(v_{i}\right)+f\left(u_{i}\right)+f\left(u_{i j-1}\right)+f\left(u_{i j}\right) \\
+f\left(u_{i j+1}\right)=1+0+0+0+0 \\
=1 .
\end{gathered}
$$

For $i=1$, if $v_{1} \in P_{n}$ then

$$
\begin{aligned}
\sum_{u \in N\left[v_{1}\right]} f(u)=f\left(v_{1}\right) & +f\left(v_{2}\right)+f\left(u_{1}\right)+f\left(u_{11}\right)+f\left(u_{12}\right) \\
& +\cdots+f\left(u_{1 m}\right) \\
& =1+1+0+0+0+\cdots+0=2 .
\end{aligned}
$$

If $u_{1} \in W_{1, m}$ then

$$
\begin{gathered}
\sum_{u \in N\left[u_{1}\right]} f(u)=f\left(v_{1}\right)+f\left(u_{1}\right)+f\left(u_{11}\right)+f\left(u_{12}\right)+\cdots \\
+f\left(u_{1 m}\right)=1+0+0+0+\cdots+0 \\
=1 .
\end{gathered}
$$

If $u_{1 j} \in W_{1, m}$ then

$$
\begin{gathered}
\sum_{u \in N\left[u_{1 j}\right]} f(u)=f\left(v_{1}\right)+f\left(u_{1}\right)+f\left(u_{1 j-1}\right)+f\left(u_{1 \mathrm{j}}\right) \\
+f\left(u_{1 j+1}\right)=1+0+0+0+0 \\
=1 .
\end{gathered}
$$

Similar is the case for $i=n$.

That is $f$ is satisfying the conditions of a unidominating function and hence it follows that $f$ is a unidominating function.

$$
\text { Now } f(V)=\sum_{u \in P_{n}} f(u)+\sum_{u \in W_{1, m}} f(u)
$$

$$
\begin{aligned}
& =\underbrace{(1+1+\cdots+1)}_{(n-\text { times })}+ \\
& \underbrace{\{\underbrace{(0+0+\cdots+0)}_{(1+m-\text { times })}+\underbrace{(0+0+\cdots+0)}_{\left(1+m-{ }^{2}\right.}+\cdots+\underbrace{(0+0+\cdots+0)}_{(1+m-\text { times })}\}}_{(n \text {-times })} \\
& =n .
\end{aligned}
$$

Therefore $f(V)=n$.

Case 2: Define a function $f: V \rightarrow\{0,1\}$ by

$f\left(v_{i}\right)=0$ for $i=1,2, \ldots \ldots, n$,

$f\left(u_{i}\right)=1$ for $i=1,2, \ldots \ldots, n$ and $f\left(u_{i j}\right)=0$ for $i=1,2, \ldots \ldots, n$ and $j=1,2, \ldots \ldots, m$.

Now we verify that $f$ is a unidominating function.

Let $i \neq 1$ and $i \neq n$.

If $v_{i} \in P_{n}$ then

$$
\begin{aligned}
& \sum_{u \in N\left[v_{i}\right]} f(u)=f\left(v_{i-1}\right)+f\left(v_{i}\right)+f\left(v_{i+1}\right)+f\left(u_{i}\right)+f\left(u_{i 1}\right) \\
&+ f\left(u_{i 2}\right)+\cdots+f\left(u_{i m}\right) \\
&=0+0+0+1+0+0+\cdots+0=1 .
\end{aligned}
$$

If $u_{i} \in W_{1, m}$ then

$$
\begin{aligned}
\sum_{u \in N\left[\mathrm{u}_{i}\right]} f(u)=f\left(v_{i}\right) & +f\left(u_{i}\right)+f\left(u_{i 1}\right)+f\left(u_{i 2}\right)+\cdots \\
& +f\left(u_{i m}\right)=0+1+0+0+\cdots+0 \\
& =1 .
\end{aligned}
$$

If $u_{i j} \in W_{1, m}$ then

$$
\begin{gathered}
\sum_{u \in N\left[u_{i j}\right]} f(u)=f\left(v_{i}\right)+f\left(u_{i}\right)+f\left(u_{i j-1}\right)+f\left(u_{i j}\right) \\
+f\left(u_{i j+1}\right)=0+1+0+0+0 \\
=1 .
\end{gathered}
$$

As in Case 1 we can verify in similar lines for $i=1$ and $i=n$.

Thus $f$ becomes a unidominating function.

$$
\begin{aligned}
& \text { Now } f(V)=\sum_{u \in P_{n}} f(u)+\sum_{u \in W_{1, m}} f(u) \\
& =\underbrace{(0+0+\cdots+0)}_{(n-\text { times })} \\
& +\underbrace{\{\underbrace{(1+0+\cdots+0)}_{(1+m-\text { times })}+\underbrace{(1+0)}_{(1+m-\text { times })}+\cdots+\underbrace{(1+0+\cdots+0)}_{(1+m-\text { times })}\}}_{(n-\text { times })} \\
& =n \text {. }
\end{aligned}
$$

Therefore $f(V)=n$.

Now for all other possibilities of assigning values 0 and 1 to the vertices $v_{i}$ of $P_{n}$ and vertex $u_{i}$ and vertices $u_{i j}$ in each copy of $W_{1, m}$, we can see that the resulting functions are not unidominating functions.

Therefore the functions defined in Case 1 and Case 2 are the only two functions which are unidominating functions.

As $f(V)=n$ for these two functions it follows that $\gamma_{u}\left(P_{n} \odot W_{1, m}\right)=n$.

Theorem 3.2: The number of unidominating functions of $P_{n} \odot W_{1, m}$ with minimum weight $n$ is 2 .

Proof: Follows by Theorem 3.1.

\section{UPPER UNIDOMINATION NUMBER OF $P_{n} \odot W_{1, m}$}

In this section the concepts of minimal unidominating function, upper unidomination number are defined. The upper unidomination number and the number of minimal unidominating functions of maximum weight of $P_{n} \odot W_{1, m}$ are determined. Further the results obtained are illustrated. 
Definition 1: Let $G(V, E)$ be a graph and $f, g$ be functions from $V$ to $\{0,1\}$. We say that $g<f$ if $g(u) \leq f(u) \forall u \in$ $V$, with strict inequality for at least one vertex $u$.

Definition 2: A unidominating function $f: V \rightarrow\{0,1\}$ is called a minimal unidominating function if for all $g<f, g$ is not a unidominating function.

Definition 3: The upper unidomination number of a graph $G(V, E)$ is defined as

$\max \{f(V) / f$ is a minimal unidominating function $\}$.

It is denoted by $\Gamma_{u}(G)$.

Theorem 4.1: The upper unidomination number of $P_{n} \odot W_{1, m}$ is $n$.

Proof: Let $P_{n} \odot W_{1, m}$ be the given corona product graph.

Case 1: Define a function $f: V \rightarrow\{0,1\}$ by

$f\left(v_{i}\right)=1$ for $i=1,2, \ldots \ldots, n$,

$f\left(u_{i}\right)=0$ for $i=1,2, \ldots \ldots, n$

and $f\left(u_{i j}\right)=0$ for $i=1,2, \ldots \ldots, n$ and $j=1,2, \ldots \ldots, m$.

This function is same as the function defined in Case 1 of Theorem 3.1 and it is shown that $f$ is a unidominating function.

Now we check for the minimality of $f$.

Define a function $g: V \rightarrow\{0,1\}$ by

$g\left(v_{i}\right)$

$=\left\{\begin{array}{l}0 \\ 1\end{array}\right.$ for $v_{i}=v_{k} \in P_{n}$ for some $k$, where $k \neq 1, k \neq n$,

$g\left(u_{i}\right)=0, u_{i} \in K_{1, m}$ for $i=1,2, \ldots \ldots, n$

and $g\left(u_{i j}\right)=0, u_{i j} \in K_{1, m}$ for $i=1,2, \ldots \ldots, n$ and $j=$ $1,2, \ldots \ldots, m$.

Then by the definition of $g$ it is obvious that $g<f$.

Suppose $i=k$.

For $v_{k} \in P_{n}$, we have $g\left(v_{k}\right)=0$ and

$$
\begin{aligned}
& \sum_{u \in N\left[v_{k}\right]} g(u)=g\left(v_{k-1}\right)+g\left(v_{k}\right)+g\left(v_{k+1}\right)+g\left(u_{k}\right) \\
&+g\left(u_{k 1}\right)+g\left(u_{k 2}\right)+\cdots+g\left(u_{k m}\right) \\
&=1+0+1+0+0+0+\cdots+0=2 \\
& \neq 1 .
\end{aligned}
$$

That is the condition for unidominating function fails at the vertex $v_{k} \in P_{n}$ for which $g\left(v_{k}\right)=0$.

So $\sum_{u \in N\left[v_{k}\right]} g(u) \neq 1$, when $g\left(v_{\mathrm{k}}\right)=0$.

Thus $g$ is not a unidominating function.

Since $g$ is defined arbitrarily, it follows that there exists no $g<f$ such that $g$ is a unidominating function.

For all other possibilities of defining a function $g<f$, we can see that $g$ is not a unidominating function.

Hence $f$ is a minimal unidominating function.

Further $f$ is the only one minimal unidominating function because any other possible assignment of values 0,1 to the vertices of $P_{n}$ and $W_{1, m}$ does not make $f$ no more a unidominating function.

Hence $f$ is a minimal unidominating function.

$$
\text { Now } f(V)=\sum_{u \in P_{n}} f(u)+\sum_{u \in W_{1, m}} f(u)
$$

$=\underbrace{(1+1+\cdots+1)}_{(n-\text { times })}$

$+\underbrace{\{\underbrace{(0+0+\cdots+0)}_{(1+m-\text { times })}+\underbrace{(0+0)}_{(1+m-\text { times })}+\cdots+\underbrace{(0+0+\cdots+0)}_{(1+m-\text { times })}\}}_{(n-\text { times })}$

$=n$.

Thus $f(V)=n$.

Now

$\max \{f(V) / f$ is a minimal unidominating function $\}=n$, because $f$ is the only one minimal unidominating function.

Therefore $\Gamma_{u}\left(P_{n} \odot W_{1, m}\right)=n$.

Case 2: Define a function $f: V \rightarrow\{0,1\}$ by

$f\left(v_{i}\right)=0$ for $i=1,2, \ldots \ldots, n$,

$f\left(u_{i}\right)=1$ for $i=1,2, \ldots \ldots, n$

and $f\left(u_{i j}\right)=0$ for $i=1,2, \ldots \ldots, n$ and $j=1,2, \ldots \ldots, m$.

This function is same as the function defined in Case 2 of Theorem 3.1 and it is shown that $f$ is a unidominating function.

Now we check for the minimality of $f$.

Define a function $g: V \rightarrow\{0,1\}$ by

$$
g\left(v_{i}\right)=0, v_{i} \in P_{n} \text { for } i=1,2, \ldots \ldots, n
$$

$$
\begin{aligned}
& g\left(u_{i}\right) \\
& =\left\{\begin{array}{l}
0 \text { for } u_{i}=u_{k} \in W_{1, m} \text { for some } k, \text { where } k \neq 1, n, \\
1
\end{array}\right. \text { otherwise, }
\end{aligned}
$$

and $g\left(u_{i j}\right)=0, u_{i j} \in W_{1, m}$

for $i=1,2, \ldots \ldots, n$ and $j=1,2, \ldots \ldots, m$.

Then by the definition of $g$ it is obvious that $g<f$.

Suppose $i=k$.

For $v_{k} \in P_{n}$ we have $g\left(u_{k}\right)=0$ and

$$
\begin{aligned}
\sum_{u \in N\left[v_{k}\right]} g(u)=g( & \left.v_{\mathrm{k}-1}\right)+g\left(v_{k}\right)+g\left(v_{k+1}\right)+g\left(u_{k}\right) \\
& +g\left(u_{k 1}\right)+g\left(u_{k 2}\right)+\cdots+g\left(u_{k m}\right) \\
& =0+0+0+0+0+0+\cdots+0=0 \\
& \neq 1
\end{aligned}
$$

That is the condition for unidominating function fails at the vertex $v_{k} \in P_{n}$ for which $g\left(u_{k}\right)=0$.

So $\sum_{u \in N\left[v_{k}\right]} g(u) \neq 1$, when $g\left(u_{\mathrm{k}}\right)=0$.

Thus $g$ is not a unidominating function.

Since $g$ is defined arbitrarily, it follows that there exists no $g<f$ such that $g$ is a unidominating function.

For all other possibilities of defining a function $g<f$, we can see that $g$ is not a unidominating function. 
Hence $f$ is a minimal unidominating function.

Further $f$ is the only one minimal unidominating function because any other possible assignment of values 0,1 to the vertices of $P_{n}$ and $W_{1, m}$ does not make $f$ no more a unidominating function.

Hence $f$ is a minimal unidominating function.

$$
\text { Now } f(V)=\sum_{u \in P_{n}} f(u)+\sum_{u \in W_{1, m}} f(u)
$$

$=$

$$
\begin{aligned}
& \underbrace{(0+0+\cdots+0)}_{(n \text {-times })}+ \\
& \underbrace{\left\{\begin{array}{c}
(1+0+\cdots+0) \\
=n .
\end{array}\right.}_{\left(\begin{array}{l}
\text {-times }) \\
(1+m \text {-times })
\end{array}\right.}
\end{aligned}
$$

Thus $f(V)=n$.

Now

$\max \{f(V) / f$ is a minimal unidominating function $\}=n$, because $f$ is the only one minimal unidominating function.

Therefore $\Gamma_{u}\left(P_{n} \odot W_{1, m}\right)=n$

Theorem 4.2: The number of minimal unidominating functions of $P_{n} \odot W_{1, m}$ with maximum weight $n$ is 2 .

Proof: Follows by Theorem 4.1 .

\section{ILLUSTRATIONS}

\section{Unidomination number}

Theorem 3.1: Case 1

The functional values are given at each vertex of the graph $P_{6} \odot W_{1,6}$.

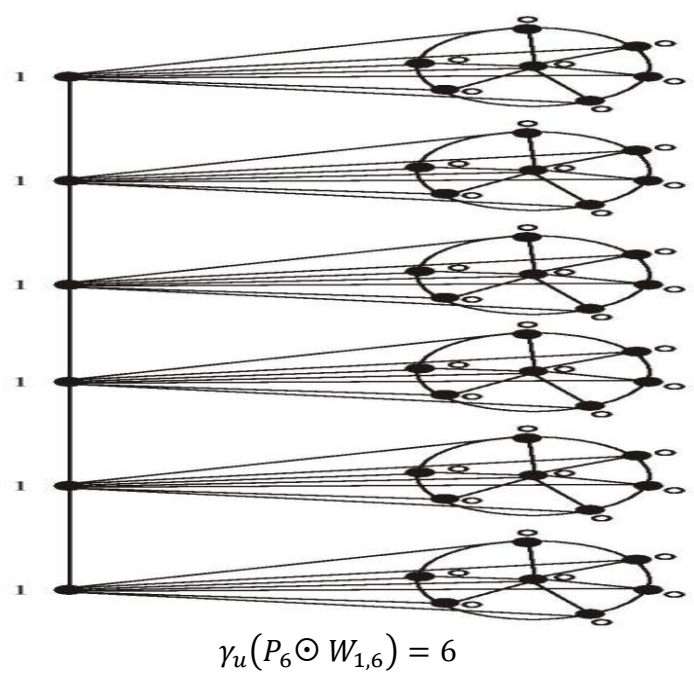

Theorem 3.1: Case 2

The functional values are given at each vertex of the graph $P_{5} \odot W_{1,6}$.

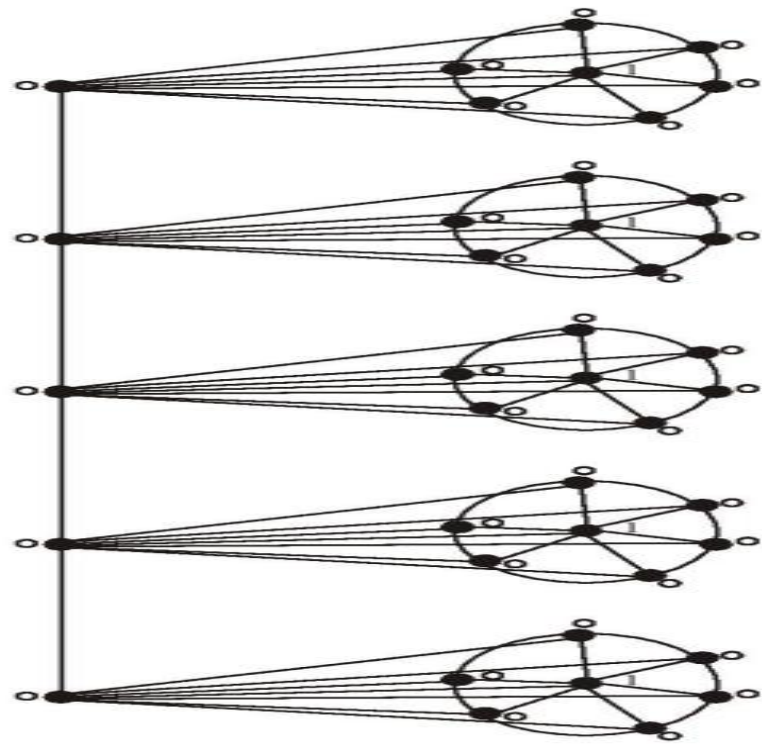

$\gamma_{u}\left(P_{5} \odot W_{1,6}\right)=6$

\section{CONCLUSION}

It is interesting to study various graph theoretic properties and domination parameters of corona product graph of a path with a wheel. Unidominating functions and upper unidomination number of this graph are studied by the authors. Study of these graphs enhances further research and throws light on future developments.

\section{REFERENCES}

[1] Anantha Lakshmi, V. A Study on Unidominating and Total Unidominating functions of Some Standard

[2] Graphs, Ph.D. thesis, Sri Padmavati Mahila Visvavidyalayam, Tirupati, Andhra Pradesh, India, (2015).

[3] Berge, C. The Theory of Graphs and its Applications, Methuen, London (1962).

[4] Frucht, R. Harary, F On the corona of Two Graphs. AequationesMathematicae, 1970,

[5] Volume 4, Issue 3, pp. 322-325

[6] Hedetniemi S .M, Hedetniemi, S.T. and Wimer, T. V. Linear time resource allocation algorithms for trees. Technical report URI - 014, Department of Mathematics, Clemson University, 1987.

[7] T.W. Haynes, T. Hedetniemi, and P.J. Slater, Fundamentals of Domination in Graphs, Marcel Dekker, New York, 1998.

[8] T.W. Haynes, S.T. Hedetniemi, and P.J. Slater, Domination in Graphs: Advanced Topics, Marcel Dekker, New York, 1998.

[9] Ore, O. Theory of Graphs, Amer. Soc. Colloq. Publ. Vol.38. Amer. Math. Soc., Providence, RI, (1962). 